\title{
The impact of school opening model on SARS-CoV-2 community incidence and mortality
}

\author{
Zeynep Ertem ${ }^{1}$, Elissa M. Schechter-Perkins ${ }^{\circledR 2}{ }^{2}$, Emily Oster ${ }^{3}$, Polly van den Berg ${ }^{4}$, Isabella Epshtein ${ }^{5}$, \\ Nathorn Chaiyakunapruk ${ }^{6}$, Fernando A. Wilson7, Eli Perencevich ${ }^{8,9}$, Warren B. P. Pettey ${ }^{6,10,11}$, \\ Westyn Branch-Elliman ${ }^{5,12,13,14 \bowtie}$ and Richard E. Nelson, $70,11,14$
}

The role that traditional and hybrid in-person schooling modes contribute to the community incidence of SARS-CoV-2 infections relative to fully remote schooling is unknown. We conducted an event study using a retrospective nationwide cohort evaluating the effect of school mode on SARS-CoV-2 cases during the 12 weeks after school opening (July-September 2020, before the Delta variant was predominant), stratified by US Census region. After controlling for case rate trends before school start, state-level mitigation measures and community activity level, SARS-CoV-2 incidence rates were not statistically different in counties with in-person learning versus remote school modes in most regions of the United States. In the South, there was a significant and sustained increase in cases per week among counties that opened in a hybrid or traditional mode versus remote, with weekly effects ranging from $9.8(95 \%$ confidence interval $(\mathrm{Cl})=2.7-16.1)$ to $21.3(95 \% \mathrm{Cl}=9.9-32.7)$ additional cases per 100,000 persons, driven by increasing cases among 0-9 year olds and adults. Schools can reopen for in-person learning without substantially increasing community case rates of SARS-CoV-2; however, the impacts are variable. Additional studies are needed to elucidate the underlying reasons for the observed regional differences more fully.

○) uring the first few months of the COVID-19 pandemic, primary and secondary schools in the United States were closed to in-person education as part of the national response to control the spread of SARS-CoV-2 (ref. ${ }^{1}$ ). This decision was guided by data extrapolated from influenza transmission models, which suggested school closures as an effective measure for reducing the basic reproductive number of respiratory viral infections $s^{1,2}$, and early evidence suggesting that non-pharmaceutical public health interventions, including school closures, were associated with improved SARS-CoV-2 outbreak control ${ }^{3,4}$.

Modeling studies and time series analyses from across the world differ in their assessment of the impact of reopening schools on community SARS-CoV-2 transmission ${ }^{5-7}$. Elementary school children are at lower risk of severe illness than other age groups and their role in driving transmission in the community is cloudy ${ }^{8,9}$. However, there are multiple close interactions between individuals from separate households in a school setting; thus, interactions that occur in schools, even if each contact is lower risk, may contribute to SARS-CoV-2 spread. If children and school staff become infected at school, these transmissions may lead to subsequent transmissions to family members and other contacts, potentially resulting in increases in community transmission of SARS-CoV-2. Recently published studies about the impact of school mode on community transmission from Indiana, Texas and other states found conflicting results ${ }^{10-12}$, with some analyses suggesting substantial increases in case rates associated with school openings, others suggesting a small impact ${ }^{13,14}$ and still others suggesting that opening schools to in-person learning has no impact on community case rates after adjusting for community incidence ${ }^{15}$ and minimal impact on hospitalization rates when COVID-19 hospitalizations within a county are kept under 36-44 per 100,000 (ref. ${ }^{16}$ ).

Thus, the association between type of school reopening mode (for example, virtual, hybrid or in-person) and community spread of SARS-CoV-2 continues to be a critical policy question. Although school closure early in the pandemic was associated with lower SARS-CoV-2 incidence ${ }^{3}$, the impact of school closures in addition to other public infection prevention measures, such as business restrictions, social distancing, masking, scaling up of testing and contact tracing is unknown. The aim of this national, retrospective cohort study was to evaluate the impact of school mode and opening to in-person education on subsequent changes in community incidence of SARS-CoV-2.

\section{Results}

A summary of the study's main findings, major limitations and policy implications, intended for nonspecialist readers is presented in Table 1. In total, 519 counties representing 1,050 school districts had a school opening mode available. After excluding the Pacific region of the West due to limited variation (59 out of 64 fully remote, 3 hybrid, 2 traditional), 459 counties consisting of 895 school districts

'Systems Science and Industrial Engineering Department, Binghamton University, State University of New York, New York, NY, USA. ${ }^{2}$ Department of Emergency Medicine, Boston University School of Medicine and Boston Medical Center, Boston, MA, USA. ${ }^{3}$ Brown University Watson Institute for International and Public Affairs, Providence, RI, USA. ${ }^{4}$ Beth Israel Deaconess Medical Center, Department of Medicine, Section of Infectious Diseases, Boston, MA, USA. ${ }^{5}$ VA Boston Center for Healthcare Organization and Implementation Research (CHOIR), Boston, MA, USA. ${ }^{6}$ Department of Pharmacotherapy, University of Utah College of Pharmacy, Salt Lake City, UT, USA. ${ }^{7}$ Matheson Center for Health Care Studies, University of Utah, Salt Lake City, UT, USA. ${ }^{8}$ Center for Access \& Delivery Research and Evaluation, lowa City VA Health Care System, lowa City, IA, USA. ${ }^{9} \mathrm{Carver}$ College of Medicine, Department of Internal Medicine, The University of lowa, lowa City, IA, USA. ${ }^{10}$ IDEAS Center, Veterans Affairs Salt Lake City Health Care System, Salt Lake City, UT, USA. "Division of Epidemiology, University of Utah School of Medicine, Salt Lake City, UT, USA. "2VA Boston Healthcare System, Department of Medicine, Section of Infectious Diseases, Boston, MA, USA. ${ }^{13}$ Department of Medicine, Harvard Medical School, Boston, MA, USA. ${ }^{14}$ These authors

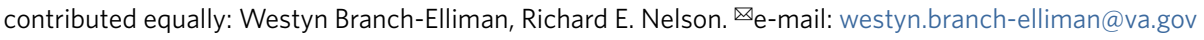




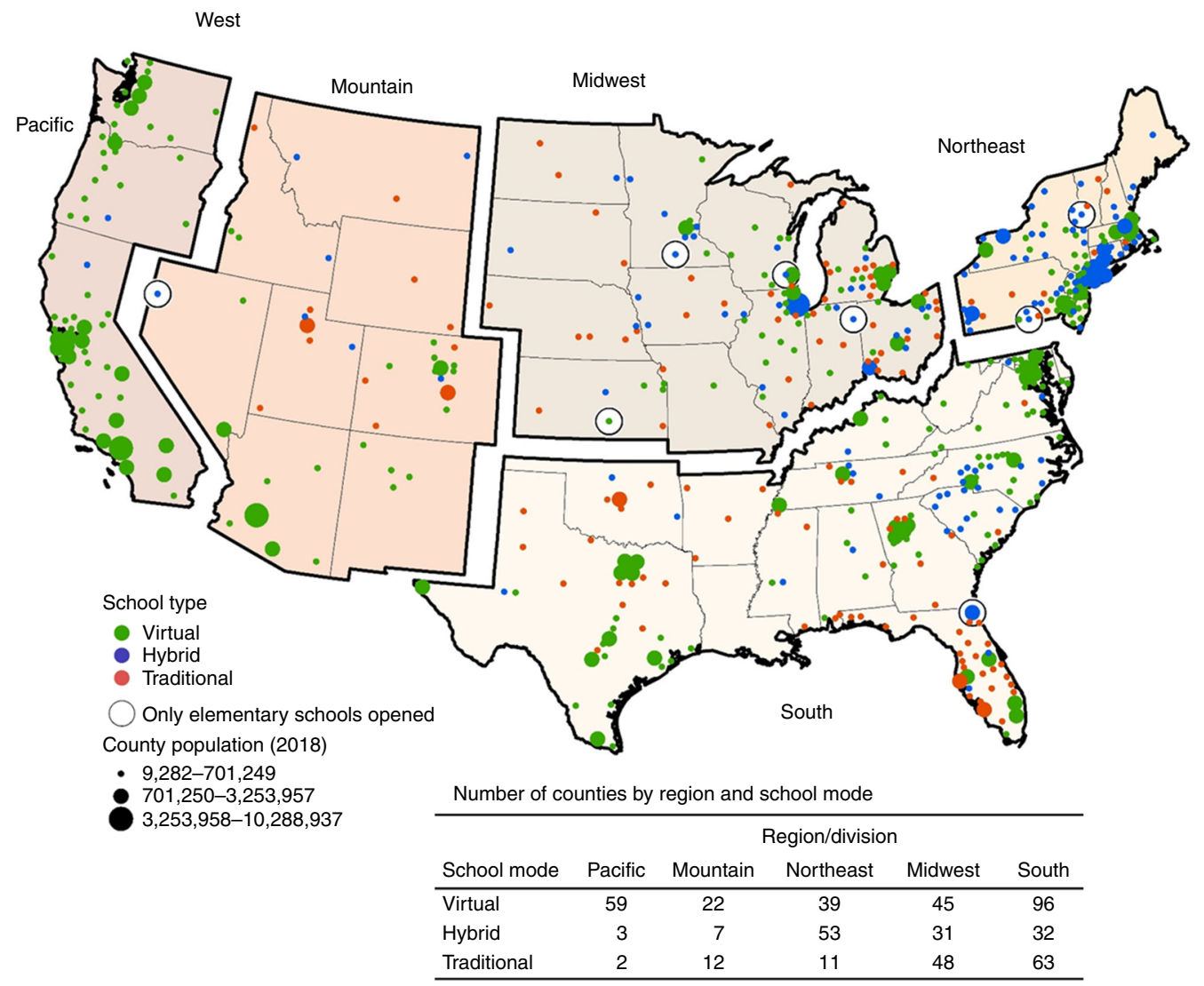

Fig. 1 | Map of counties included in the analysis. Counties with green markers opened in a fully virtual learning model, counties with blue markers opened in hybrid mode and counties with red markers opened in a fully traditional (in-person) learning mode. The size of county markers is related to county population size, with larger markers indicating larger populations. The table inset depicts the number of counties by region and school opening mode.

\begin{tabular}{|c|c|}
\hline Topic & Summary \\
\hline Background & $\begin{array}{l}\text { Little is known about the impact of in-person learning (hybrid or traditional) versus fully remote schooling on the spread of } \\
\text { SARS-CoV- } 2 \text { in the community. }\end{array}$ \\
\hline $\begin{array}{l}\text { Main findings and } \\
\text { limitations }\end{array}$ & $\begin{array}{l}\text { In most regions of the United States, we found no association between SARS-CoV- } 2 \text { community infection rates and school } \\
\text { opening mode during the initial weeks of the } 2020-2021 \text { school year. In the South, however, there was a significant and sustained } \\
\text { increase in community infection rates among counties that opened for in-person learning compared to those that were fully } \\
\text { remote, driven by cases in 0-9 year olds and adults. } \\
\text { Major limitations include that the underlying reason for regional differences could not be delineated. Additionally, the study was } \\
\text { conducted on data from the } 2020-2021 \text { school year, when the Delta variant was not in circulation and before vaccines were } \\
\text { widely available for adults and adolescents in the United States; thus, results may not be generalizable to the current school year. }\end{array}$ \\
\hline Policy implications & $\begin{array}{l}\text { Schools can reopen for in-person learning during the pandemic without substantially increasing community case rates of } \\
\text { SARS-CoV-2; however, the impacts on community transmission are variable. Additional studies are needed to elucidate the } \\
\text { reasons for the regional differences identified in our analysis more fully. }\end{array}$ \\
\hline
\end{tabular}

were included (Fig. 1). In all counties, one school mode predominated (that is, there were no counties split evenly between remote and in-person learning).

Among the included counties, 103 were in the Northeast, 41 in the Mountain division, 124 in the Midwest and 191 in the South (Extended Data Table 1). Traditional, full in-person schooling was the most common mode in the Midwest (48 out of 124); in the Northeast, hybrid learning models predominated (53 out of 103); and in the South and Mountain division, virtual learning was the most common (South, 96 out of 191, Mountain, 22 out of 41).

Initial school opening dates varied but ranged from an earliest start date of 22 July 2020 to a latest start date of 28 September
2020 (Extended Data Fig. 1). Significant demographic differences by region were identified (Extended Data Table 1). Notable differences in community activity and infection control policies identified between regions included higher rates of business closure and activity restrictions in the Northeast and Western regions, increased contact tracing in the Northeast, stricter masking policies and regulations in the Northeast and West and more access to testing in the Northeast and Midwest (Table 2). Manual review of community-level mitigation policies found that masking mandates were generally implemented earlier in the Northeast versus other regions; masking mandates were least common and tended to be implemented latest in the South (5 out of 20 Southern counties had 
Table 2 | Descriptive statistics of covariates included in the regression models

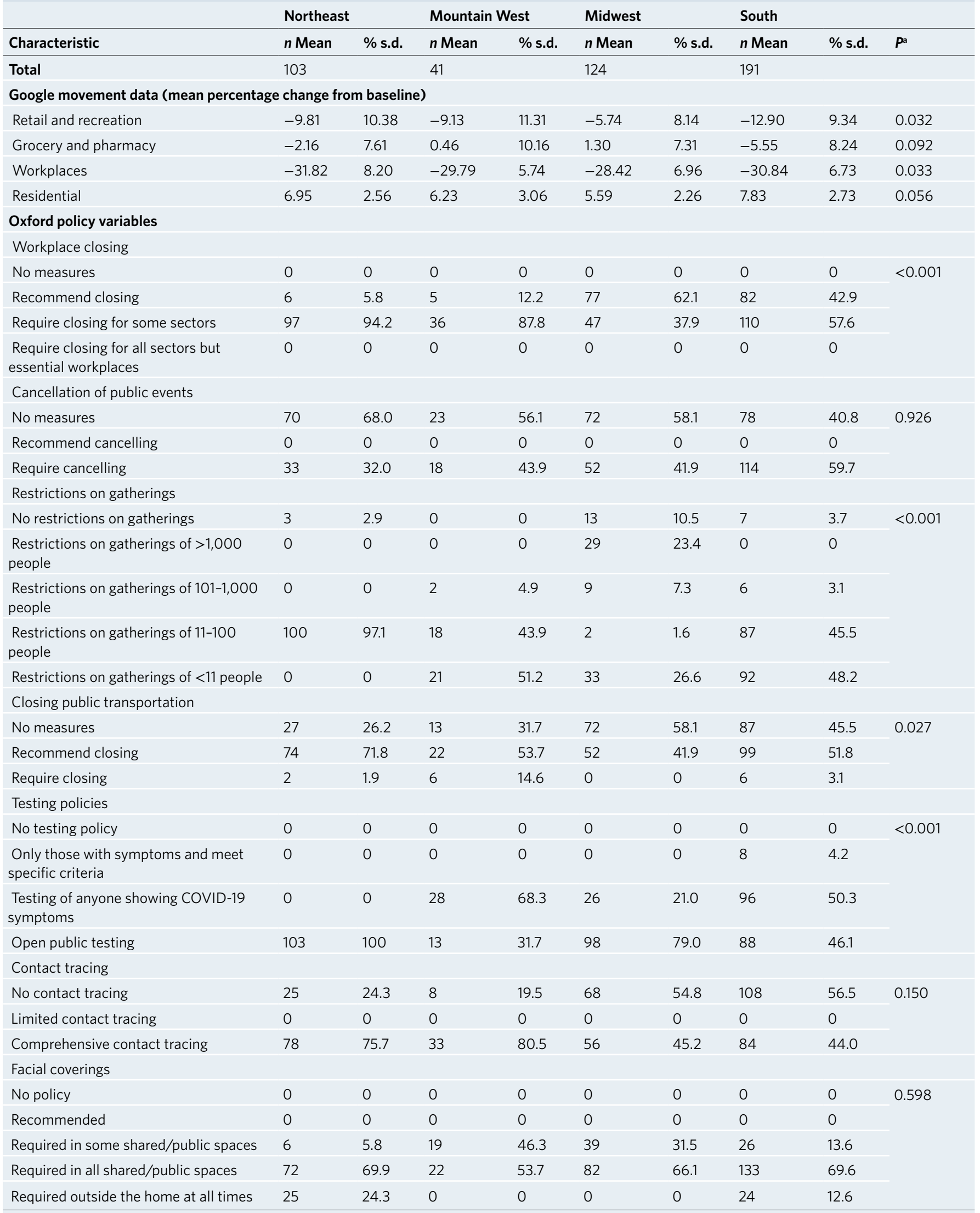

${ }^{a} P$ values were obtained from one-way analysis of variance models. 


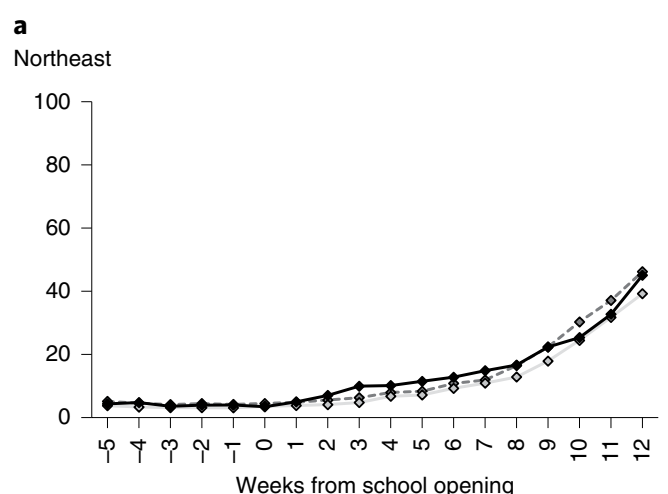

C

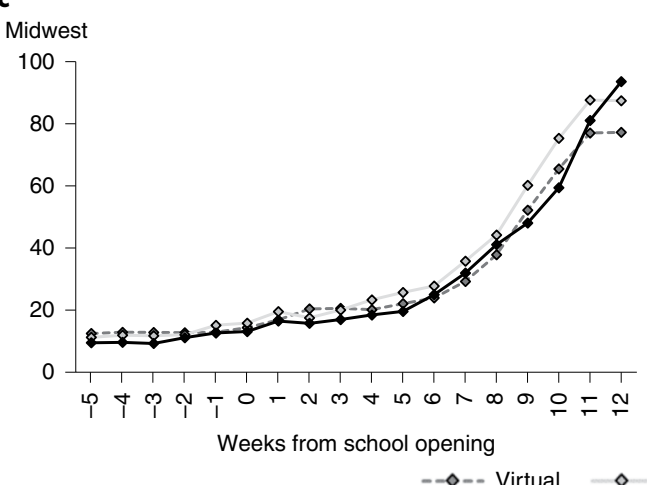

b

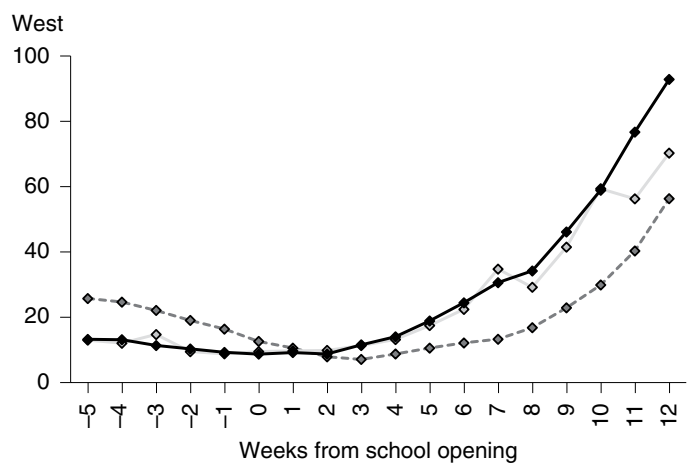

d

South

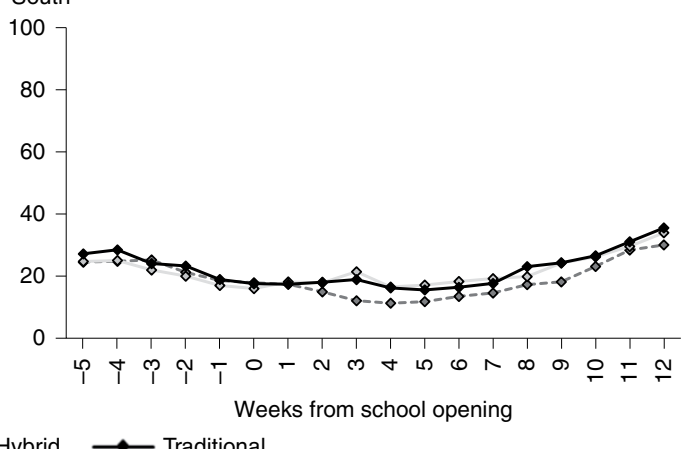

Fig. 2 | Unadjusted mean SARS-CoV-2 cases per 100,000 residents stratified by region; week 0 denotes initial school opening. a-d, The solid black lines indicate traditional school mode, the solid gray lines indicate hybrid school mode and the dashed gray lines indicate virtual school mode for the Northeast (a), West (b), Midwest (c) and South (d).

no mask mandate or a masking mandate started after school opening versus 2 out of 20 in the Midwest, 1 out of 20 in the Northeast and 0 out of 20 in the West).

Unadjusted mean SARS-CoV-2 cases per 100,000 residents per week stratified by region are shown in Fig. 2. Increasing SARS-CoV-2 case counts across all regions during the weeks after the start of school were identified, regardless of school mode. The adjusted absolute differences in SARS-CoV-2 cases, which include an accounting for baseline community prevalence before school opening, between counties with hybrid and traditional school opening modes relative to counties with virtual learning models are presented in Fig. 3. In the Northeast and Midwest regions, differences in SARS-CoV-2 case rates were not detectably different across any of the 3 learning modes, although there was a small increase in cases 6-9 weeks after school opening in the Midwest in counties with traditional learning; no increase was found in counties with hybrid learning modes. In the South, there was a statistically significant increase in cases in counties that opened for hybrid or traditional modes compared to virtual. In the West, there was an increase in cases in counties with a hybrid learning mode.

After adjustment, a traditional school mode was associated with increases in the number of SARS-CoV-2 cases compared to a fully remote mode from week 4 (effect $=13.8$ cases per 100,000 residents, $95 \% \mathrm{CI}=1.1-26.4)$ to week $6(\mathrm{effect}=11.2,95 \% \mathrm{CI}=0.1-22.3)$ in the Midwest. In the South, a traditional in-person mode was associated with increases in the number of SARS-CoV-2 cases during the period from week 2 after school opening (effect $=10.7$ cases per 100,000 residents, $95 \% \mathrm{CI}=3.6-17.8$ ) to week 12 after opening, (effect $=10.0,95 \% \mathrm{CI}=3.1-16.8$ ).

In the South, a hybrid school mode was associated with increases in the number of SARS-CoV-2 cases from week 3 (effect $=9.7,95 \%$ $\mathrm{CI}=1.5-17.9)$ to week $12(\mathrm{effect}=6.4,95 \% \mathrm{CI}=0-12.7)$. In the West, a hybrid mode was associated with increases in cases from week 5 $($ effect $=19.9,95 \% \mathrm{CI}=0.2-39.7)$ to week 12 (effect $=30.7,95 \%$ $\mathrm{CI}=3.4-58.1)$. There was no impact of school opening mode on subsequent COVID-19-related deaths during the entire 12-week period after school opening in any region (Fig. 4 and Extended Data Fig. 2).

Sensitivity analyses using $P<0.01$ or $P<0.000347$ as a threshold for statistical significance yielded similar results in the South but the impact of a hybrid learning mode in the West was nonsignificant after adjustment for multiple testing (Extended Data Figs. 3 and 4).

In the adjusted models, the impact of school opening mode on SARS-CoV-2 cases stratified by age group varied between and within regions (Extended Data Figs. 5-7). Across all regions, there were no differences in 10-19 year olds. Case increases associated with in-person learning in the South and Midwest were driven by increases in cases diagnosed in $\geq 20$ year olds. In the South, there was a statistically significant and sustained increase in cases among 0-9 year olds during the $2-10$ week period after school opening.

\section{Discussion}

This national cohort study, which included nearly half of all public school student enrollment across the United States, found regional variation on the impact of school reopening policy on the community incidence of SARS-CoV-2. In the South, which tended to have more limited community-level mitigation measures and which opened during a period with relatively high community prevalence of SARS-CoV-2 cases, reopening schools for in-person learning (using either a hybrid or traditional approach) was associated with a subsequent sustained increase in community case rates of SARS-CoV-2, driven by case increases among adults and children under the age of ten. The effect of school mode in the South was robust to both sensitivity analyses with stringent cutoffs for statistical significance. In the West, opening in a hybrid school mode was 

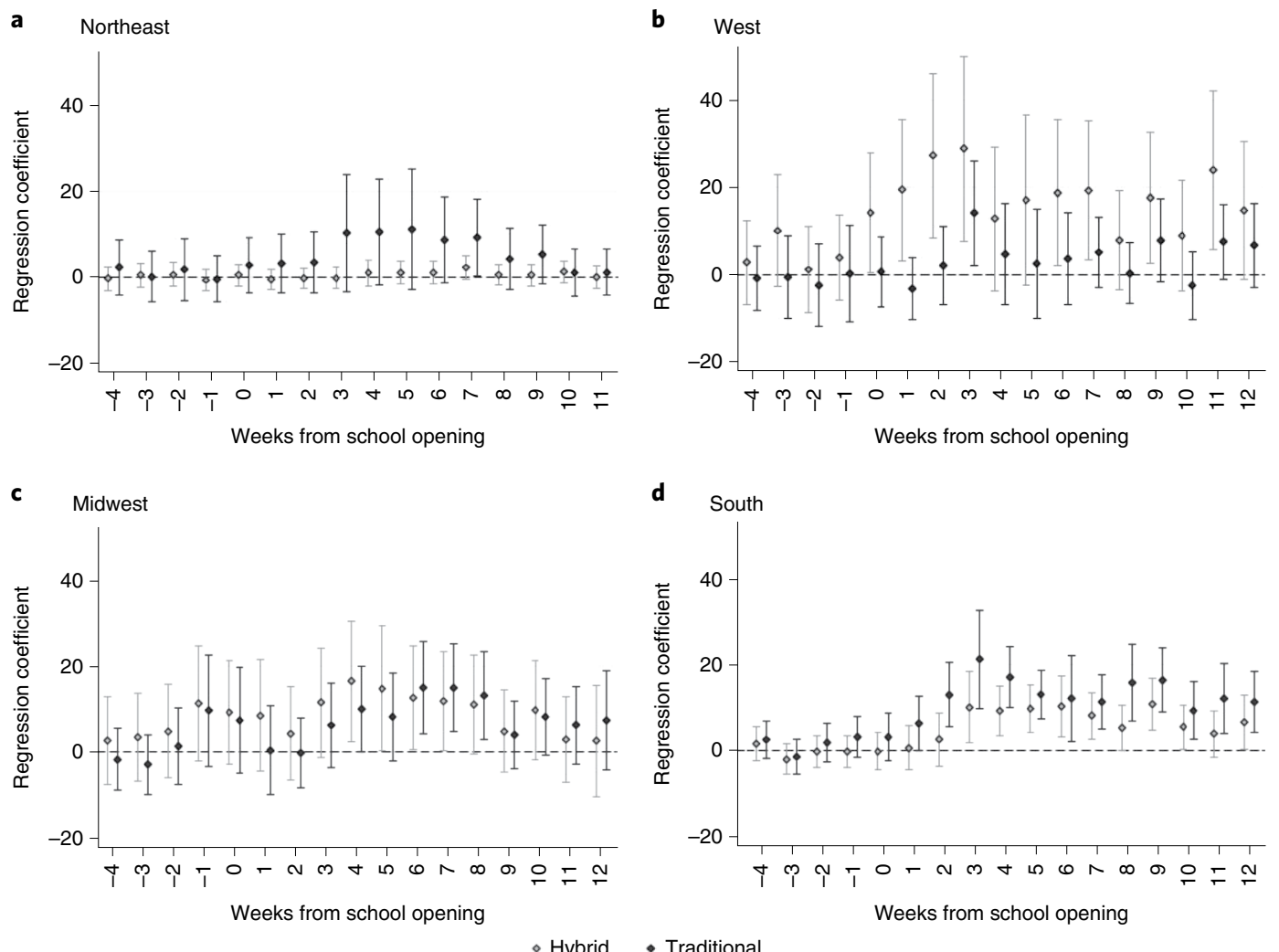

Fig. 3 | Adjusted absolute difference between SARS-CoV-2 cases in counties with hybrid and traditional school modes relative to virtual for each week, with week $\mathbf{O}$ being the week when school started for each county, stratified by region. a-d, Results were generated from multivariate Poisson regressions with robust s.e. for the Northeast (a), West (b), Midwest (c) and South (d). The black markers indicate traditional school mode and the gray markers indicate hybrid school mode. The whiskers for each marker depict the upper and lower bounds of the $95 \% \mathrm{Cl}(n=1,512$ for the Northeast, 648 for the West, 1,887 for the Midwest and 3,110 for the South). Google mobility data were not available for week 12 following school opening in the Northeast, thus results are presented for the 11 weeks following school opening in that region (a).

associated with sustained increased community case rates; however, these findings were not statistically significant in the sensitivity analyses. In other regions, where adoption of community public health measures were more substantial and where schools opened during times of relatively low prevalence, we found no impact of school opening mode on subsequent community incidence of SARS-CoV-2. These data add to a growing body of literature about the impact of school opening policy on SARS-CoV-2 transmission and public health measures for pandemic control ${ }^{13,14,16,17}$.

Although evidence demonstrates that children, particularly elementary school children, are at low risk of severe COVID-19 (ref. ${ }^{18}$ ), data are mixed about the role children may play in household and community transmission of SARS-CoV-2 (refs. ${ }^{16,19,20}$ ). Our nationwide study adds to a growing body of data about the role that in-person learning plays in SARS-CoV-2 transmission in the surrounding community and is consistent with previous studies supporting broad infection prevention strategies for SARS-CoV-2 control. Additionally, our study demonstrates that, while school opening can be associated with increases in case rates in some regions, notably those that opened during times of peak SARS-CoV-2 in the community, those case increases may not translate to detectable increases in COVID-19 mortality. Policy decisions about closing schools for in-person learning must be weighed against the harms of ongoing school closures.

In our dataset, the most extreme and sustained increase in SARS-CoV-2 cases associated with school opening was in the South, where school opening was associated with a weekly increase in cases ranging from 9.8 to 21.3 per 100,000 people. Our observational study cannot fully explain the reason for the different effects identified in the South; however, in this region, infection control measures both inside and outside of school were limited. In regions with more substantive infection control efforts both inside of school settings and in the broader communities, such as the Northeast, there was no increase in community case incidence associated with opening schools and a trend toward a decrease in SARS-CoV-2 cases among children after schools opened for in-person instruction. Additional potentially contributing factors include that schools in the South opened when cases of SARS-CoV-2 in the community were relatively high, raising the question of whether the impact of school opening on community transmission rates is dependent on community prevalence, which would be consistent with the finding by Harris et al. ${ }^{16}$ that the impact of school mode on COVID-19 is dependent on SARS-CoV-2 hospitalization rates in the community. Additionally, it is possible that different climactic conditions may play a role in the trajectory of cases, independent of school mode policy. Thus, it is possible that there is a differential effect of in-person schooling on community transmission that is dependent on a variety of factors, such as mitigation strategies in schools and in surrounding communities, different weather and humidity patterns in the South compared to other regions or higher cases in the community leading to more case introductions in schools and ultimately more community transmission originating inside schools ${ }^{16}$. Additional studies are needed to delineate the impacts of these different factors more fully.

Our study adds to a growing body of literature about the impact of school closures as a policy measure for reducing the basic 

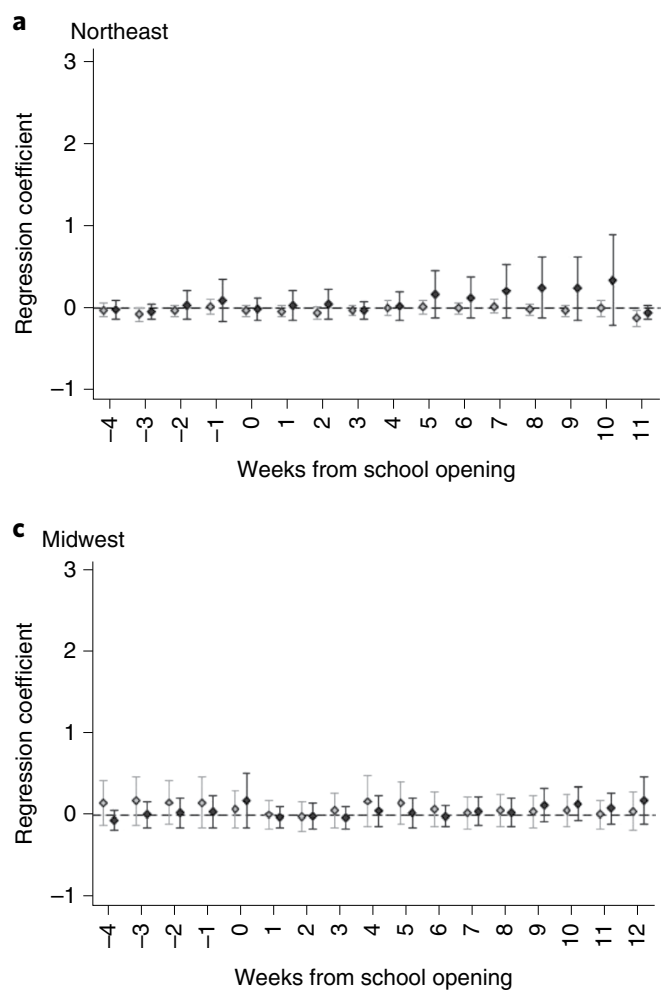

- Hybrid
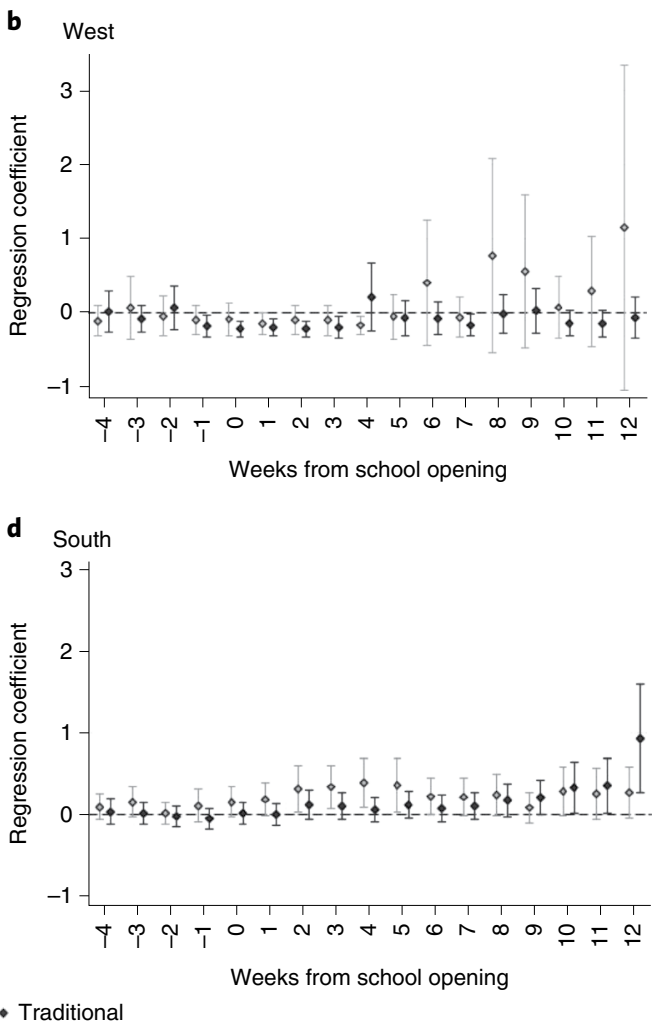

Fig. 4 | Adjusted absolute difference between SARS-CoV-2 deaths in counties with hybrid and traditional school modes relative to virtual for each week, with week 0 being the week when school started for each county, stratified by region. a-d, Results for the Northeast (a), West (b), Midwest (c) and South (d) were generated from multivariate Poisson regressions with robust s.e. The black markers indicate a traditional school mode and the gray markers indicate a hybrid school mode. The whiskers for each marker depict the upper and lower bounds of the $95 \% \mathrm{Cl}$ ( $n=1,512$ for the Northeast, 648 for the West, 1,887 for the Midwest and 3,110 for the South). Google mobility data were not available for week 12 following school opening in the Northeast, thus results are presented for the 11 weeks following school opening in that region (a).

reproductive number of SARS-CoV-2. Early reports suggested that school closures were associated with reductions in COVID-19 deaths, although study authors acknowledged that, due to the simultaneous implementation of a variety of public health measures, the impact of school closure specifically could not be delineated fully $\mathrm{y}^{3,5,7,11,12}$. Other investigations found conflicting results, with some suggesting that opening schools is associated with an increase in SARS-CoV-2 cases in the community and others suggesting minimal or no impact ${ }^{13-16}$. A meta-analysis found that studies with the lowest risk of statistical bias did not find a substantial impact of school mode on community incidence ${ }^{5,17}$. A recent study evaluating the impact of school mode on community SARS-CoV-2 cases in Texas found substantial increases associated with reopening schools for in-person learning ${ }^{11}$. However, the Texas study, unlike ours, did not control for temporal trends occurring before the start of school. Across all regions, school opening occurred in a background of increasing case counts; thus, controlling for temporal trends and other county-level factors is critical for isolating the impact of school mode from other simultaneous events that may occur at the same time as changes in school learning modes.

Multiple previous studies conducted in the setting of multifaceted infection control plans demonstrated low rates of transmission in urban, suburban and rural public school settings ${ }^{21-25}$. Conversely, SARS-CoV-2 school clusters reported in the United States and around the world highlight that substantial in-school transmission can occur under some circumstances. A recent national survey found that, among students attending schools that adopted few or no mitigation measures, living with a student attending in-person school was associated with a higher risk of COVID-19 among family members ${ }^{26}$. However, the same study also found that the elevated risk was eliminated with the addition of more in-school mitigation measures, findings similar to those of other studies ${ }^{7,27,28}$.

Our study has several limitations. It was observational in nature and thus we are not able to determine causality, only association. The study was conducted using data collected from the 2020-2021 school year, when the more transmissible Delta variant was not the predominant strain and when vaccines were not available to adults or adolescents. Thus, it is not clear how the results of this study should be contextualized, given current transmission dynamics and vaccine availability for some public school students. Data regarding SARS-CoV-2 cases are available from the Centers for Disease Control and Prevention (CDC) stratified by decade of life but not by year of life. This has two major impacts on our study. First, cases in infants and toddlers were included in case counts for children under ten; thus, it is possible that the impact found in this age group associated with school opening was not driven by children attending elementary schools but by younger children. Second, we were not able to break down cases for middle and high school children separately. Third, given the high rates of mild and asymptomatic cases in children, it is possible that cases in these populations potentially attributed to attendance of in-person learning were not detected.

Detailed district infection control plans were not available; thus, we were not able to measure the effectiveness of specific infection prevention measures within schools on community incidence of cases. However, the aim of this study was to evaluate the impact of school opening policy on community transmission, not to address the related but distinct question of SARS-CoV-2 transmission and prevention in schools. Elementary school children appear to spread 
less than children in older grades and may be less able to participate in remote learning ${ }^{29}$. Thus, one proposed policy option was offering traditional instruction to younger children while offering hybrid or remote options to middle and high school students. Due to the very high correlation between learning modes at each of the three grade levels, we were not able to address the potential impact of this strategy on community spread of SARS-CoV-2 since only eight counties in our dataset opted for this policy model. We may not have been able to fully control for community infection prevention measures that may have impacted estimates. However, we attempted to mitigate the effect of this confounding by stratifying our analysis by region, which was highly correlated with school opening date, school opening policy and state-level infection control interventions, and by using a variety of data sources to address community response in a variety of different ways, including the Oxford dataset, which included detailed information about SARS-CoV-2 mitigation strategies, and the Google COVID-19 Community Mobility Reports, which reflected county-level activity, and by manually reviewing a subset of the counties to ensure data quality and accuracy. The use of multiple robust datasets with manual validation to ensure accuracy is a major strength of the study. Data about school mode were available at the district level and other measures were available at a county or state level; it is possible that the conversion from district to county data may have introduced bias into our findings. However, Burbio's validation data found that school opening mode is highly clustered and estimated a margin of error of $2.7 \%$ in their dataset ${ }^{30}$; this small margin of error would not have changed our study's principal findings. Finally, we could not account for private schools; however, approximately $90 \%$ of elementary and secondary school children attend public schools; thus, the impact of these missing data is likely to be small.

The association between school opening mode and county incidence of SARS-CoV-2 varies by region and may be correlated with community infection prevention measures or community incidence of SARS-CoV-2 cases at the time of school opening, both of which may also be correlated with the implementation of in-school mitigation and community mitigation measures. Although results varied by region, these findings suggest that schools can open for in-person learning during the pandemic with minimal contribution to sustained community incidence of infections, provided other public safety measures are adopted.

\section{Online content}

Any methods, additional references, Nature Research reporting summaries, source data, extended data, supplementary information, acknowledgements, peer review information; details of author contributions and competing interests; and statements of data and code availability are available at https://doi.org/10.1038/ s41591-021-01563-8.

Received: 13 July 2021; Accepted: 28 September 2021; Published online: 27 October 2021

\section{References}

1. Viner, R. M. et al. School closure and management practices during coronavirus outbreaks including COVID-19: a rapid systematic review. Lancet Child Adolesc. Health 4, 397-404 (2020).

2. Jackson, C., Vynnycky, E., Hawker, J., Olowokure, B. \& Mangtani, P. School closures and influenza: systematic review of epidemiological studies. BMJ Open 3, e002149 (2013).

3. Auger, K. A. et al. Association between statewide school closure and COVID-19 incidence and mortality in the US. JAMA 324, 859-870 (2020).

4. Pan, A. et al. Association of public health interventions with the epidemiology of the COVID-19 outbreak in Wuhan, China. JAMA 323 , 1915-1923 (2020).

5. Haug, N. et al. Ranking the effectiveness of worldwide COVID-19 government interventions. Nat. Hum. Behav. 4, 1303-1312 (2020).
6. Naimark, D. et al. Simulation-based estimation of SARS-CoV-2 infections associated with school closures and community-based nonpharmaceutical interventions in Ontario, Canada. JAMA Netw. Open 4, e213793 (2021).

7. Lee, B., Hanley, J. P., Nowak, S., Bates, J. H. T. \& Hébert-Dufresne, L. Modeling the impact of school reopening on SARS-CoV-2 transmission using contact structure data from Shanghai. BMC Public Health 20, 1713 (2020)

8. $\mathrm{Li}, \mathrm{X}$. et al. The role of children in transmission of SARS-CoV-2: a rapid review. J. Glob. Health 10, 011101 (2020).

9. Ludvigsson, J. F. Children are unlikely to be the main drivers of the COVID-19 pandemic-a systematic review. Acta Paediatr. 109, 1525-1530 (2020)

10. Bosslet, G. T. et al. The effect of in-person primary and secondary school instruction on county-level SARS-CoV-2 spread in Indiana. Clin. Infect. Dis. https://doi.org/10.1093/cid/ciab306 (2021).

11. Courtemanche, C. J., Le, A. H., Yelowitz, A. \& Zimmer, R. School Reopenings, Mobility, and COVID-19 Spread: Evidence from Texas (National Bureau of Economic Research, 2021).

12. España, G. et al. Impacts of K-12 school reopening on the COVID-19 epidemic in Indiana, USA. Epidemics 37, 100487 (2021).

13. Bravata, D., Cantor, J. H., Sood, N. \& Whaley, C. M. Back to School: The Effect of School Visits During COVID-19 on COVID-19 Transmission (National Bureau of Economic Research, 2021).

14. Vlachos, J., Hertegård, E. \& Svaleryd, H. B. The effects of school closures on SARS-CoV-2 among parents and teachers. Proc. Natl Acad. Sci. USA 118, e2020834118 (2021).

15. Goldhaber, D. et al. To What Extent Does In-Person Schooling Contribute to the Spread of COVID-19? Evidence from Michigan and Washington (National Bureau of Economic Research, 2021).

16. Harris, D. N., Ziedan, E. \& Hassig, S. The Effects of School Reopenings on COVID-19 Hospitalizations (National Center for Research on Education Access and Choice, 2021).

17. Walsh, S. et al. Do school closures and school reopenings affect community transmission of COVID-19? A systematic review of observational studies.BMJ Open 11, e053371 (2021).

18. Castagnoli, R. et al. Severe acute respiratory syndrome coronavirus 2 (SARS-CoV-2) infection in children and adolescents: a systematic review. JAMA Pediatr. 174, 882-889 (2020).

19. Kim, J. et al. Role of children in household transmission of COVID-19. Arch. Dis. Child. 106, 709-711 (2021).

20. Grijalva, C. G. et al. Transmission of SARS-COV-2 infections in householdsTennessee and Wisconsin, April-September 2020. MMWR Morb. Mortal. Wkly. Rep. 69, 1631-1634 (2020).

21. van den Berg, P. et al. Effectiveness of three versus six feet of physical distancing for controlling spread of COVID-19 among primary and secondary students and staff: a retrospective, state-wide cohort study. Clin. Infect. Dis. https://doi.org/10.1093/cid/ciab230 (2021).

22. Falk, A. et al. COVID-19 cases and transmission in $17 \mathrm{~K}-12$ schools-Wood County, Wisconsin, August 31-November 29, 2020. MMWR Morb. Mortal. Wkly. Rep. 70, 136-140 (2021).

23. Hershow, R. B. et al. Low SARS-CoV-2 transmission in elementary schools-Salt Lake County, Utah, December 3, 2020-January 31, 2021. MMWR Morb. Mortal. Wkly. Rep. 70, 442-448 (2021).

24. Dawson, P. et al. Pilot investigation of SARS-CoV-2 secondary transmission in kindergarten through grade 12 schools implementing mitigation strategies-St. Louis County and city of Springfield, Missouri, December 2020. MMWR Morb. Mortal. Wkly. Rep. 70, 449-455 (2021).

25. Varma, J. K. et al. COVID-19 infections among students and staff in New York City public schools. Pediatrics 147, e2021050605 (2021).

26. Lessler, J. et al. Household COVID-19 risk and in-person schooling. Science 372, 1092-1097 (2021).

27. Gold, J. A. W. et al. Clusters of SARS-CoV-2 infection among elementary school educators and students in one school district-Georgia, December 2020-January 2021. MMWR Morb. Mortal. Wkly. Rep. 70, 289-292 (2021)

28. Stein-Zamir, C. et al. A large COVID-19 outbreak in a high school 10 days after schools' reopening, Israel, May 2020. Euro Surveill. 25, 2001352 (2020)

29. Alonso, S. et al. Age-dependency of the propagation rate of coronavirus disease 2019 inside school bubble groups in Catalonia, Spain. Pediatr. Infect. Dis. J. https://doi.org/10.1097/INF.0000000000003279 (2021).

30. Burbio's Methodology (Burbio, 2021); https://about.burbio.com/methodology/ (2021).

Publisher's note Springer Nature remains neutral with regard to jurisdictional claims in published maps and institutional affiliations.

This is a U.S. government work and not under copyright protection in the U.S.; foreign copyright protection may apply 2021, corrected publication 2022 


\section{Methods}

To measure the impact of school mode on the community transmission of SARS-CoV-2, we created a retrospective cohort of school districts including the period immediately preceding and following school reopening in the United States (July-September 2020; Extended Data Fig. 1). Using multiple data sources, a longitudinal dataset at the county-week level was created and SARS-CoV-2 cases were examined. Variation in school opening date and mode were exploited to estimate the effect of initial school mode on SARS-CoV-2 cases and COVID-19 deaths. Data spanning the time from 5 weeks before official school opening in any of three modes (for example, traditional, hybrid, virtual) to 12 weeks after the start of school were included. County fixed effects were included to control for trends in case counts before school opening. In each of our statistical analyses, week 0 corresponds to the week when school began in that county.

Data sources. School model. School reopening mode data were obtained from Burbio, which includes manually validated information from 1,200 school districts across the United States, representing approximately 35,000 schools in 50 states, and $47 \%$ of student enrollment in public K-12 schools ${ }^{30}$. Districts are classified into type of school mode, including traditional, defined as students participating in in-person learning $\geq 4 \mathrm{~d}$ per week; hybrid, defined as students divided into cohorts and attending school in-person 2-3 d per week; and virtual, defined as students attending school in a fully remote mode with no live, in-person instruction. Data available in the Burbio dataset include the date the school district opened and the proportion of schools that opened in each of the three different learning modes, stratified by school type (for example, elementary, defined as kindergarten to 5 th grade, middle school, defined as 6 th to $8^{\text {th }}$ grade and high school, defined as 9 th to 12th grade). To convert these school district-level data to the county level, we first took the average school mode proportion among sampled districts within a county across the three grade levels. We then assigned the school mode for the county based on the maximum value of these averaged grade level school modes; for example, if $75 \%$ of the districts within a county were hybrid, then the entire county was considered hybrid.

Community incidence and COVID-19-related deaths. Incident cases of SARS-CoV-2 per day at a county level were obtained from the CDC dataset ${ }^{31}$. Data available through these sources included daily cases, decade of age and deaths by county, starting from January 2020 (refs. ${ }^{6,32}$ ). Per CDC guidelines, both confirmed and probable cases and COVID-19 deaths were included. Daily incidence was converted into a weekly incidence for cases and deaths. The denominator for the outcome measures was the estimated number of residents in the year 2020 for each county by the US Census Bureau.

Community-level COVID mitigation measures. Data about community-level mitigation measures were obtained through the Oxford University dataset, which contains data about federal, state and substate policies ${ }^{33}$. To validate these data, a sample of districts ( $n=20$ in each of the 4 census regions) underwent manual review for the presence and type of community-level masking policy to ensure accuracy of the variables and provide insight into how state and substate mitigation measures may have differed from county-level interventions.

Community mobility data. Community mobility data were accessed from the Google COVID-19 Community Mobility Reports dataset ${ }^{34}$. These reports contain aggregated and anonymized user data through Google's location history. User data were organized into trends over time by geography and separated into various locations. The Community Mobility data provide insight into the mobility response to COVID-19 mitigation policies ${ }^{34}$. These variables are measured as the percentage change in the time individuals spent in different locations relative to a baseline time period (3 January 2020-6 February 2020).

Independent variables. The key independent variables were the county school mode, dummy variables for each week and the interaction between school mode and week variables. Our analyses controlled for important covariates to minimize confounding bias in the relationship between school mode opening and outcomes. These covariates included variables from the Google Community Mobility data (retail and recreation, grocery and pharmacy, workplaces and residential) and from the Oxford policy data (workplace closings, cancelling of public events, restrictions on gatherings, closing of public transportation, COVID testing policies, COVID contact tracing and requirements to wear masks outside the home). In addition, due to the hierarchical and longitudinal nature of our data, we included county, state, week and state-week fixed effects to control for temporal trends among other county-level factors. We chose to use a fixed effects approach as opposed to a random effects or multilevel approach because of the strict assumption that random effects be uncorrelated with other independent variables included in the model. Given regional variation and correlation within regions regarding the timing of school opening, SARS-CoV-2 case counts, county infection control strategies and school mode, the cohort was stratified by US Census region (for example, Northeast, West, Midwest, South). The Pacific division was excluded due to near uniform school mode (virtual; Fig. 1); therefore, the West region includes only the Mountain division.
Outcome variables. The primary outcome variable was change in county-level incidence of SARS-CoV-2 cases per 100,000 residents during the 12-week period after school opening to estimate any sustained impact on community spread of SARS-CoV-2 associated with in-person learning modes. Secondary outcome variables included change in COVID-19 mortality per 100,000 residents and change in incident diagnoses stratified by decade of life (0-9 years, $10-19$ years and $20+$ years), which was examined to determine if the school model was associated with increases in children and adolescents attending primary and secondary school or if the primary impact was on infections diagnosed in adults.

Data analysis. We used an event study framework ${ }^{35}$ with data from before and after K-12 schools opened for the 2020-2021 school year, before the Delta variant was predominant and before vaccine availability. Event studies are a commonly used $^{36-41}$ extension to the standard difference in differences approach. They can be exploited to estimate the effect of the occurrence of an event on an outcome over time while taking advantage of variation in the timing of exposure to this event across groups ${ }^{42}$. In our case, the week of school opening varied from early August to late September 2020 (Extended Data Fig. 1). We estimated the effect of school opening mode on SARS-CoV-2 diagnoses and COVID-19 mortality outcomes using a multivariate Poisson regression with robust standard errors. Given the strong association between region and mitigation measures in the community and timing of school opening, models were estimated separately for each of the four regions. We report the results from these models from the school mode-week interaction terms as marginal effects that are interpreted as the adjusted absolute effect of school mode per week on the outcome. Running analyses for multiple regions raised concerns about multiple hypothesis testing; to address this, we completed a sensitivity analysis using $P<0.01$ as the cutoff for statistical significance. Additionally, to account for the analysis calculated on a weekly basis, rather than in aggregate, a second sensitivity analysis, applying a Bonferroni-adjusted $P=0.000373$ for significance, was conducted. All analyses were completed using STATA v.16.

Reporting Summary. Further information on research design is available in the Nature Research Reporting Summary linked to this article.

\section{Data availability}

Data supporting this manuscript were collected from several sources. COVID-19 Community Mobility Report data from Google and the COVID-19 Government Response Tracker data from Oxford University are publicly available for download. Data from Burbio are available via purchase and data use agreements with the company. Data from the CDC restricted access dataset are available via a data use agreement with the CDC. To comply with the data use agreements with the CDC and Burbio, a datafile with random county identifiers has been posted in the Harvard Dataverse (https://dataverse.harvard.edu/dataverse/ school-opening-covid).

\section{Code availability}

The code and meta-data supporting this analysis have been posted in the Harvard Dataverse.

\section{References}

31. COVID-19 Case Surveillance Restricted Access Detailed Data (US Centers for Disease Control and Prevention., 2021); https://data.cdc.gov/ Case-Surveillance/COVID-19-Case-Surveillance-Restricted-Access-Detai/ $\mathrm{mbd} 7-\mathrm{r} 32 \mathrm{t}$

32. What is Case Surveillance? (US Centers for Disease Control and Prevention, 2021); https://wwwn.cdc.gov/nndss/datacollection.html\#: :Text=It\%20is\%20 mandatory\%20that\%20reportable,control\%20and\%20prevention $\% 20$ is $\% 20$ needed

33. Hallas, L. et al. Variation in US states' responses to COVID-19_3.0. Github https://github.com/OxCGRT/USA-covid-policy (2020).

34. COVID-19 Community Mobility Reports (Google, 2021); https://www.google. $\mathrm{com} /$ covid19/mobility

35. Khullar, D. \& Jena, A. B. Natural experiments in health care research. JAMA Health Forum 2, e210290 (2021).

36. Bellou, A. \& Bhatt, R. Reducing underage alcohol and tobacco use: evidence from the introduction of vertical identification cards. J. Health Econ. 32, 353-366 (2013).

37. Anderson, D. M., Rees, D. I. \& Sabia, J. J. Medical marijuana laws and suicides by gender and age. Am. J. Public Health 104, 2369-2376 (2014).

38. Bitler, M. P. \& Carpenter, C. S. Health insurance mandates, mammography, and breast cancer diagnoses. Am. Econ. J. Econ. Policy 8, 39-68 (2016).

39. Simon, D. Does early life exposure to cigarette smoke permanently harm childhood welfare? Evidence from cigarette tax hikes. Am. Econ. J. Appl. Econ. 8, 128-159 (2016).

40. Marcus, J. \& Siedler, T. Reducing binge drinking? The effect of a ban on late-night off-premise alcohol sales on alcohol-related hospital stays in Germany. J. Public Econ. 123, 55-77 (2015). 
41. Paik, M., Black, B. S. \& Hyman, D. A. The receding tide of medical malpractice litigation. Part 2: effect of damage caps. J. Empir. Leg. Stud. 10, 639-669 (2013).

42. Wing, C., Simon, K. \& Bello-Gomez, R. A. Designing difference in difference studies: best practices for public health policy research. Annu. Rev. Public Health 39, 453-469 (2018).

\section{Acknowledgements}

Support for this study was provided by a National Institutes of Health grant no. 1K12HL138049-01 (to W.B.-E.). The views expressed are those of the study authors and do not imply endorsement from the US Federal Government.

\section{Author contributions}

Z.E., E.M.S.-P., E.O., P.V.D.B., I.E., N.C., F.A.W., E.P., W.B.-E. and R.E.N. designed the study. Z.E., R.E.N., E.O. and W.B.P.P. created the cohort and analyzed the data. W.B.-E., Z.E., R.E.N., P.V.D.B. and I.E. wrote the manuscript. Z.E., E.M.S.-P., E.O., P.V.D.B., I.E., N.C., F.A.W., E.P., W.B.P.P., W.B.-E. and R.E.N. reviewed and edited the manuscript.

\section{Competing interests}

W.B.E. is the site principal investigator for a study funded by Gilead (funds to institution). E.M.S.-P. receives funds from the Gilead's FOCUS program. The other authors declare no competing interests.

\section{Additional information}

Extended data is available for this paper at https://doi.org/10.1038/s41591-021-01563-8.

Supplementary information The online version contains supplementary material available at https://doi.org/10.1038/s41591-021-01563-8.

Correspondence and requests for materials should be addressed to Westyn Branch-Elliman.

Reprints and permissions information is available at www.nature.com/reprints.

Nature Medicine thanks Chris Illingworth, Carlos del Rio and the other, anonymous, reviewer(s) for their contribution to the peer review of this work. Jennifer Sargent and Joao Monteiro were the primary editors on this article and managed its editorial process and peer review in collaboration with the rest of the editorial team. 


\section{$\square \mathrm{NE} \square \mathrm{W} \square \mathrm{MW} \square \mathrm{S}$}

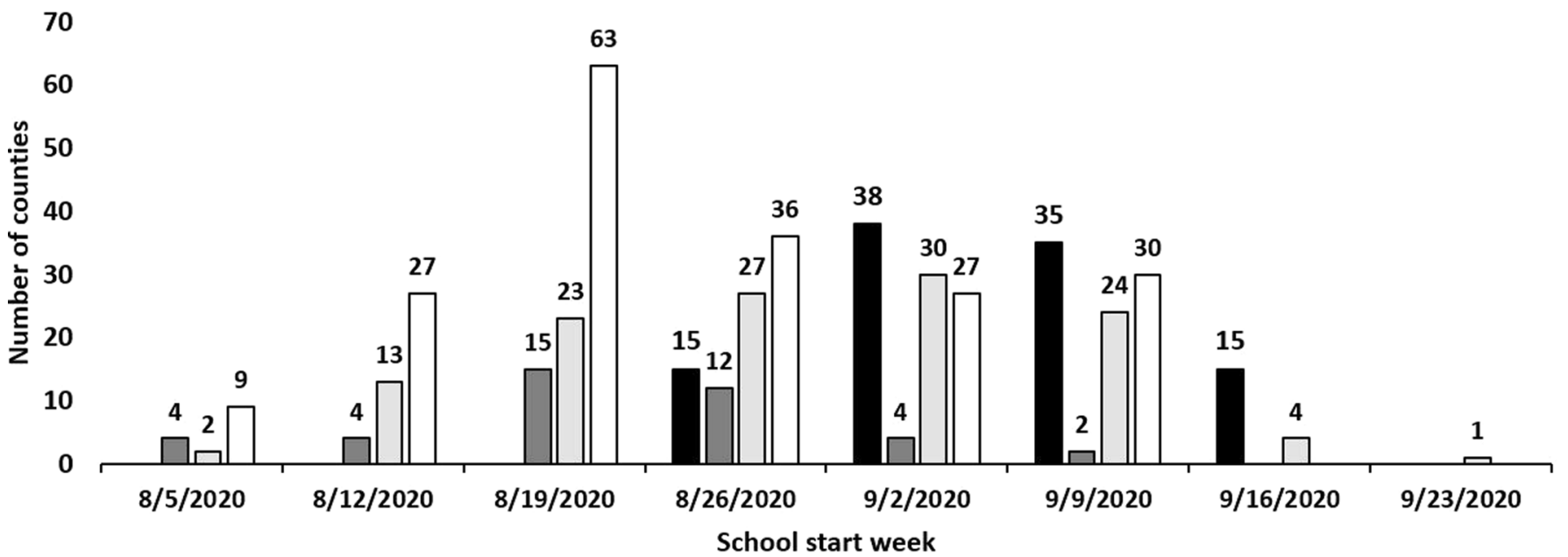

Extended Data Fig. 1 | Frequency of school start dates by region. 


\section{(a) Northeast}

1

0.8

0.6

0.4

0.2

0

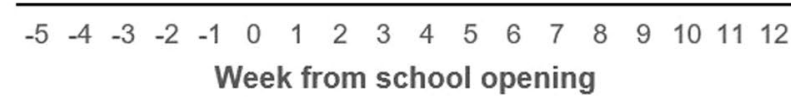

(c) Midwest

1

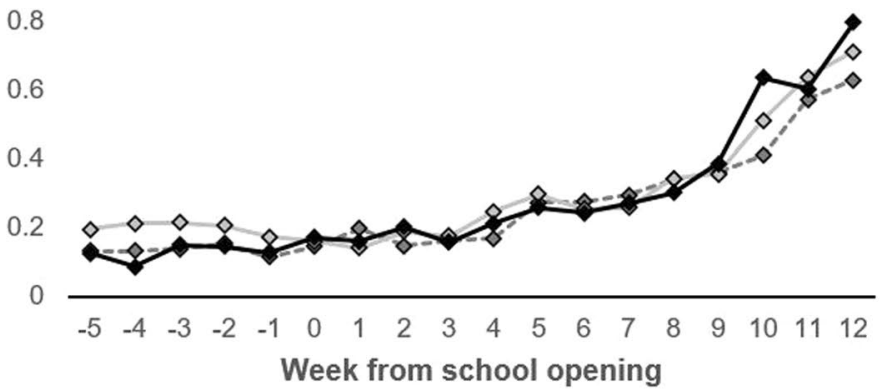

(b) West

1

0.8

0.6

0.4

0.2

0

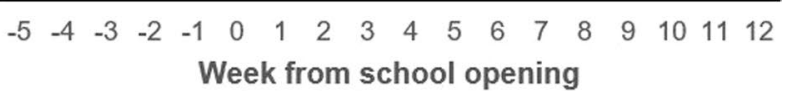

(d) South

1

0.8

0.6

0.4

0.2

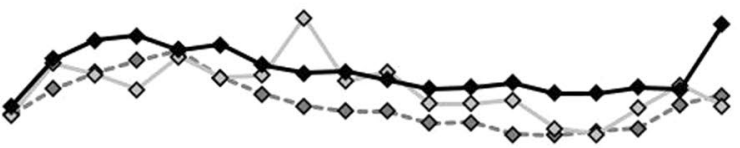

0

$\begin{array}{llllllllllllllllll}-5 & -4 & -3 & -2 & -1 & 0 & 1 & 2 & 3 & 4 & 5 & 6 & 7 & 8 & 9 & 10 & 11 & 12\end{array}$ Week from school opening

\section{$-\infty-$-Virtual $\diamond$ Hybrid $\rightarrow$ Traditional}

Extended Data Fig. 2 | Unadjusted mean SARS-CoV-2 deaths per 100,000 residents, stratified by region. Week zero denotes initial school opening. Solid black lines indicate traditional school mode, solid gray lines indicate hybrid school mode, and dashed gray lines indicate virtual school mode. 


\section{(a) Northeast}

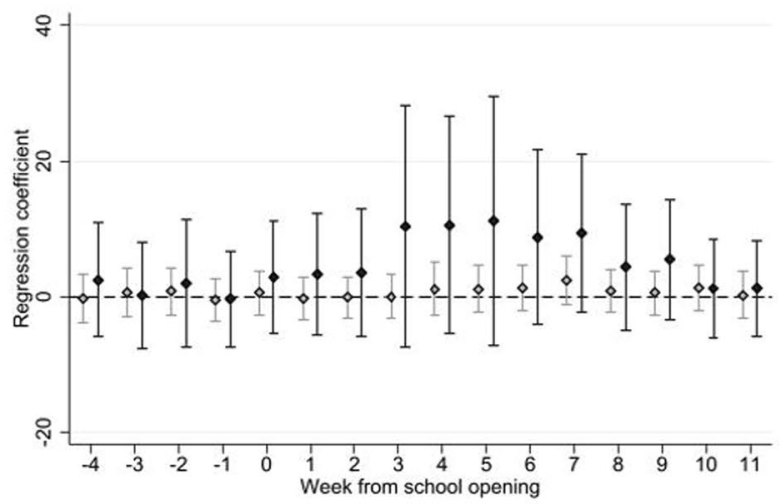

(c) Midwest

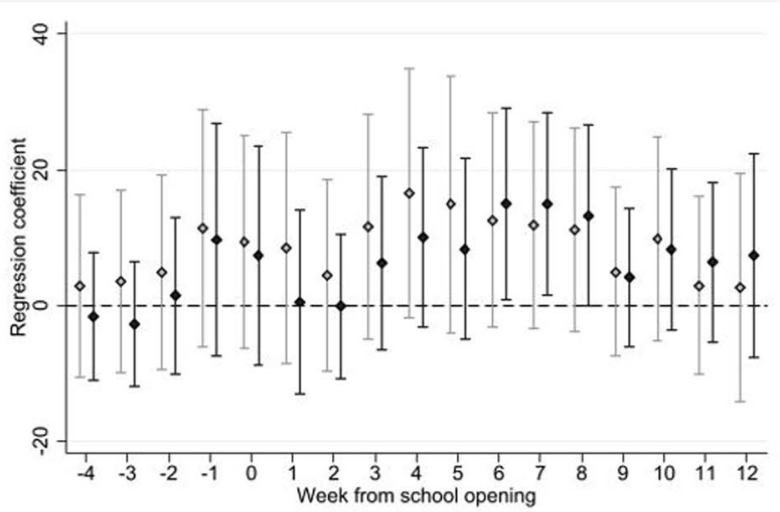

\section{(b) West}

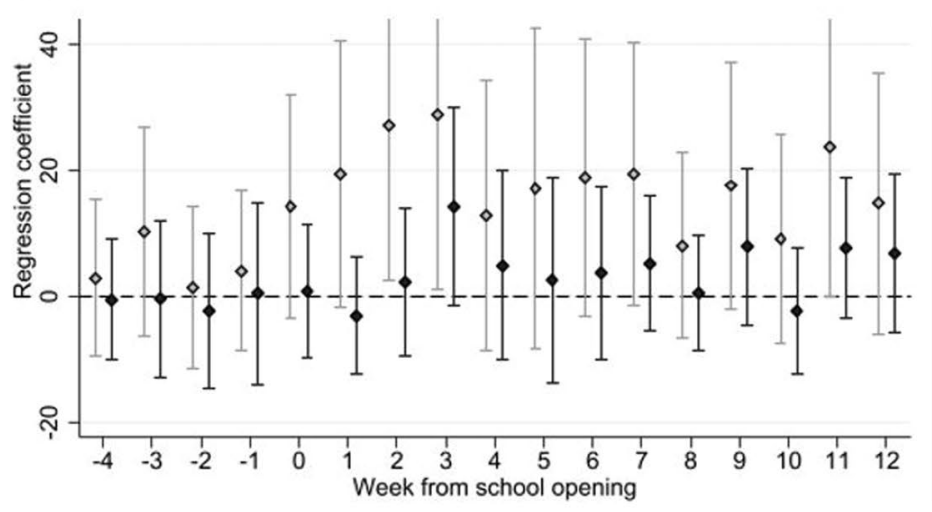

(d) South

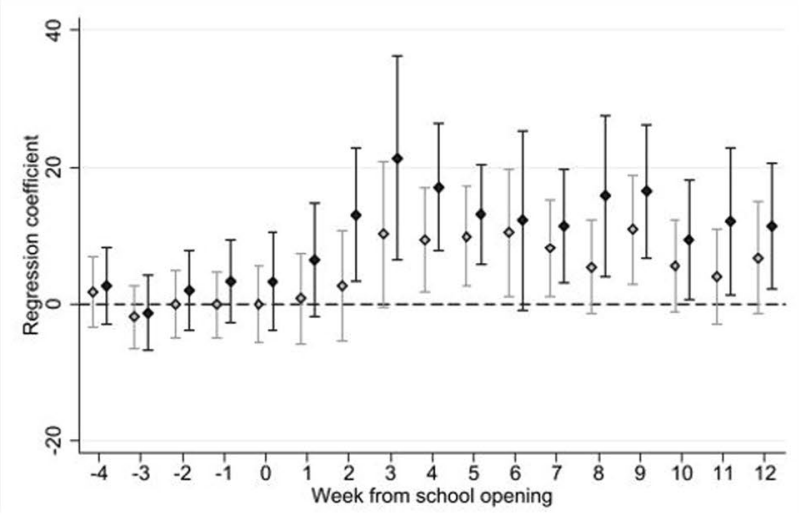

$\diamond$ Hybrid $\bullet$ Traditional

Extended Data Fig. 3 | Adjusted absolute difference between SARS-CoV-2 cases in counties with hybrid and traditional school modes relative to virtual for each week. Week 0 is the week in which school started for each county, stratified by region. Results were generated from multivariable Poisson regressions with robust standard errors. Black markers indicate traditional school mode and gray markers indicate hybrid school mode. We adjusted for multiple comparisons by using a p-value of 0.01 for statistical significance. Whiskers for each marker depict the upper and lower bounds of the $99 \%$ confidence interval ( $N=1,512$ for $N E$, 648 for $W, 1,887$ for $M W$, and 3,110 for S). Google mobility data were not available for Week 12 following school opening in the Northeast, thus results are presented for the 11 weeks following school opening in that region (panel A). 
(a) Northeast

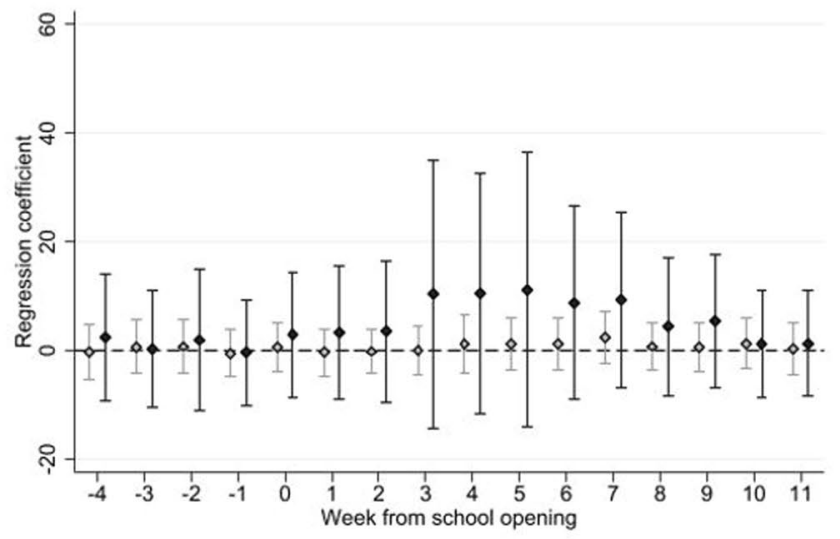

(c) Midwest

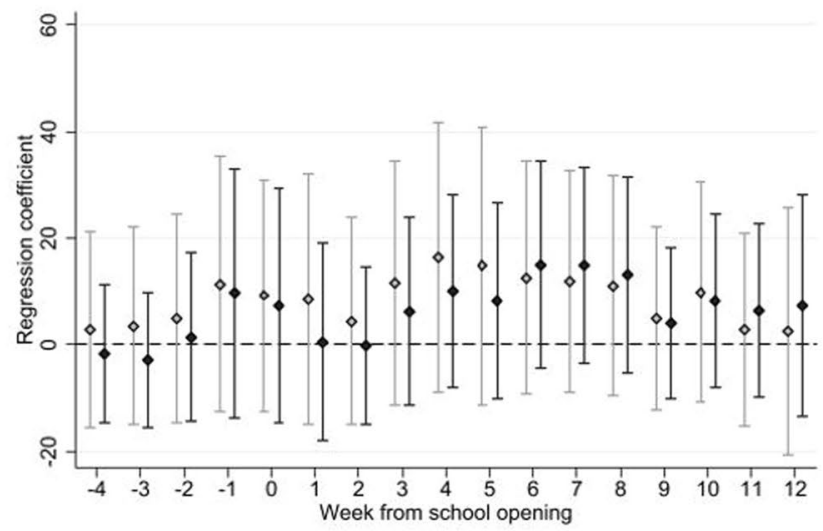

(b) West

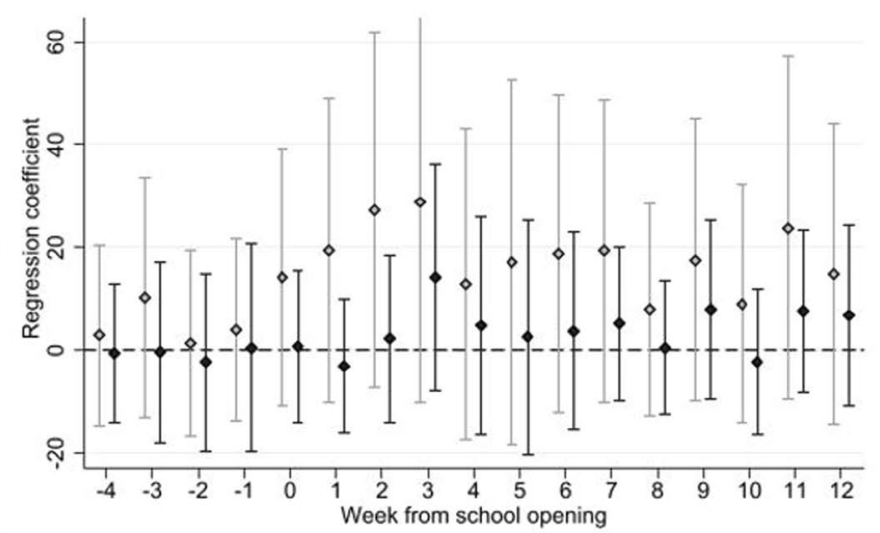

(d) South

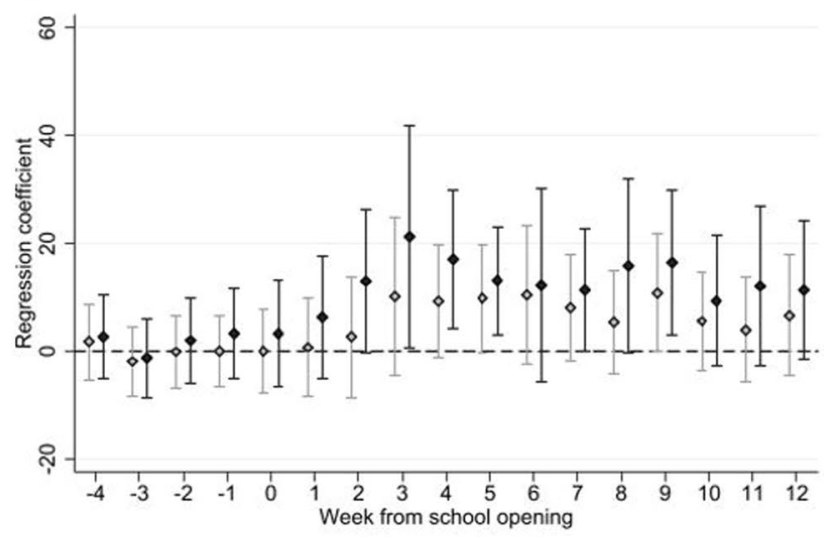

Hybrid Traditional

Extended Data Fig. 4 | Adjusted absolute difference between SARS-CoV-2 cases in counties with hybrid and traditional school modes relative to virtual for each week. Week 0 is the week in which school started for each county, stratified by region. Results were generated from multivariable Poisson regressions with robust standard errors. Black markers indicate traditional school mode and gray markers indicate hybrid school mode. We adjusted for multiple comparisons by using a p-value of 0.000373 for statistical significance. Whiskers for each marker depict the upper and lower bounds of the 99.97\% confidence interval ( $N=1,512$ for $N E, 648$ for W, 1,887 for $\mathrm{MW}$, and 3,110 for S). Google mobility data were not available for Week 12 following school opening in the Northeast, thus results are presented for the 11 weeks following school opening in that region (panel A). 
(a) Northeast

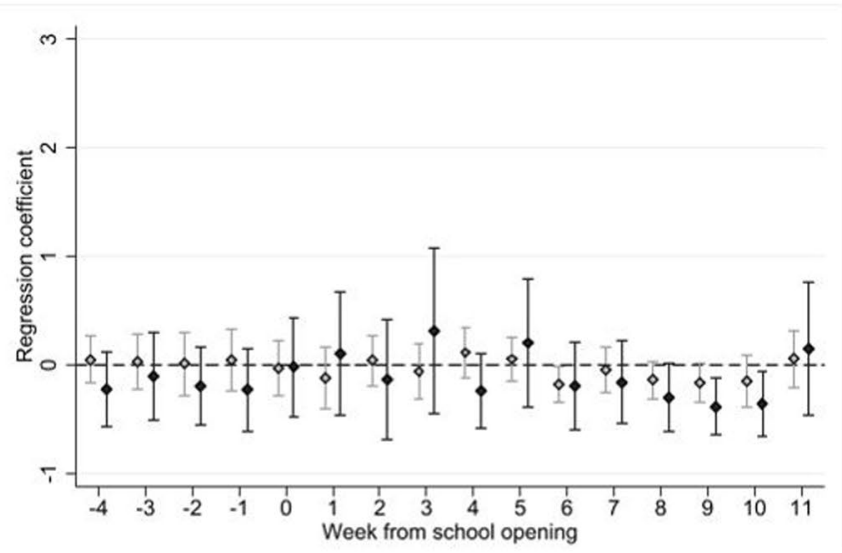

(b) Midwest

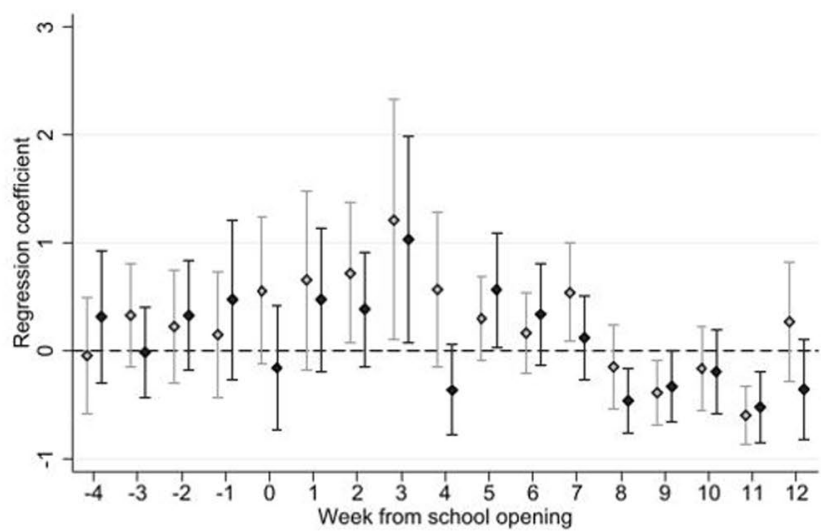

(b) West

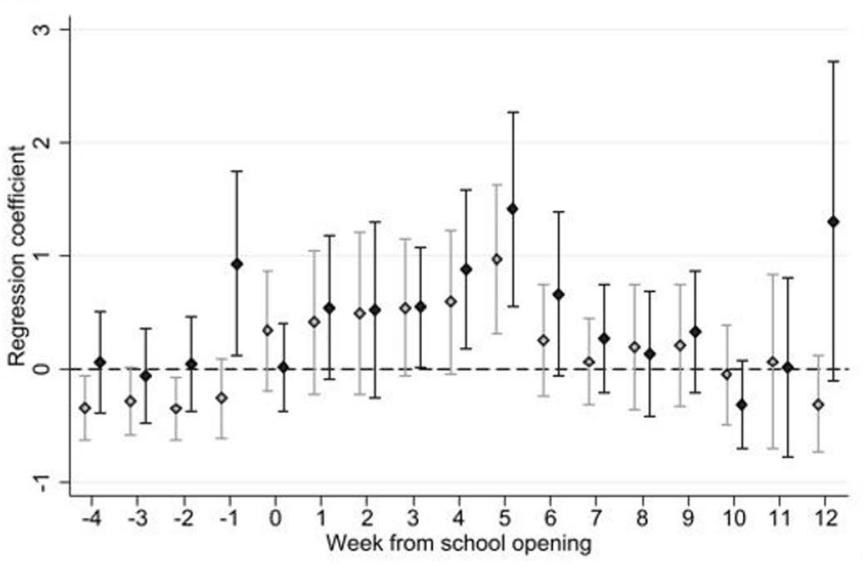

(d) South

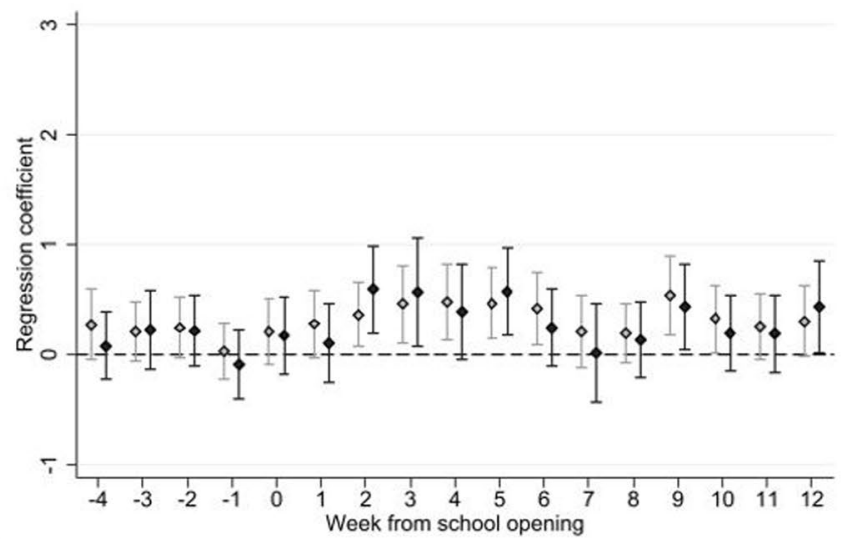

$\diamond$ Hybrid Traditional

Extended Data Fig. 5 | Adjusted absolute difference between SARS-CoV-2 cases among 0-9 year olds in counties with hybrid and traditional school modes relative to virtual for each week. Week 0 is the week in which school started for each county, stratified by region, restricted to individuals age $0-9$. Results were generated from multivariable Poisson regressions with robust standard errors. Black markers indicate traditional school mode and gray markers indicate hybrid school mode. Whiskers for each marker depict the upper and lower bounds of the $95 \%$ confidence interval ( $N=1,273$ for $N E, 567$ for W, 1,622 for MW, and 2,380 for S). Google mobility data were not available for Week 12 following school opening in the Northeast, thus results are presented for the 11 weeks following school opening in that region (panel A). 
(a) Northeast

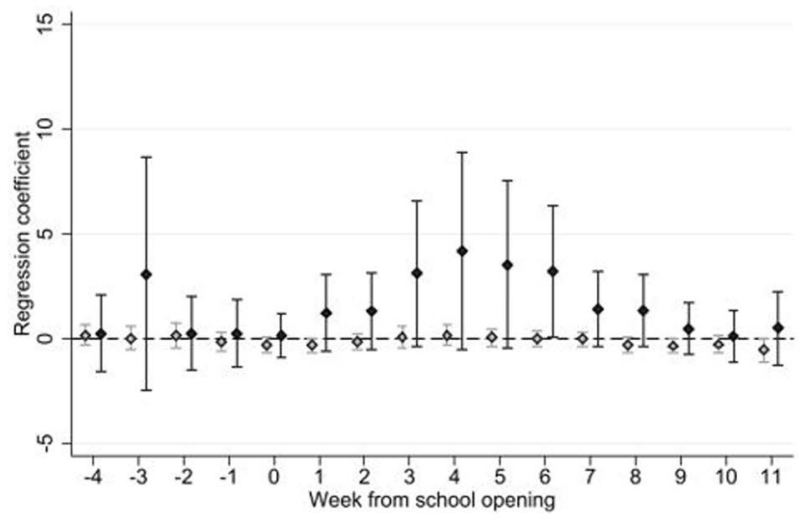

(c) Midwest

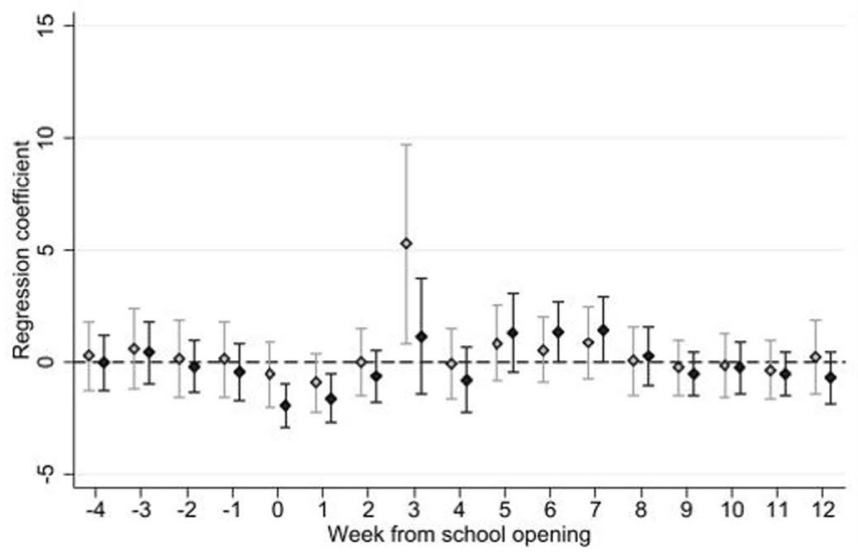

(b) West

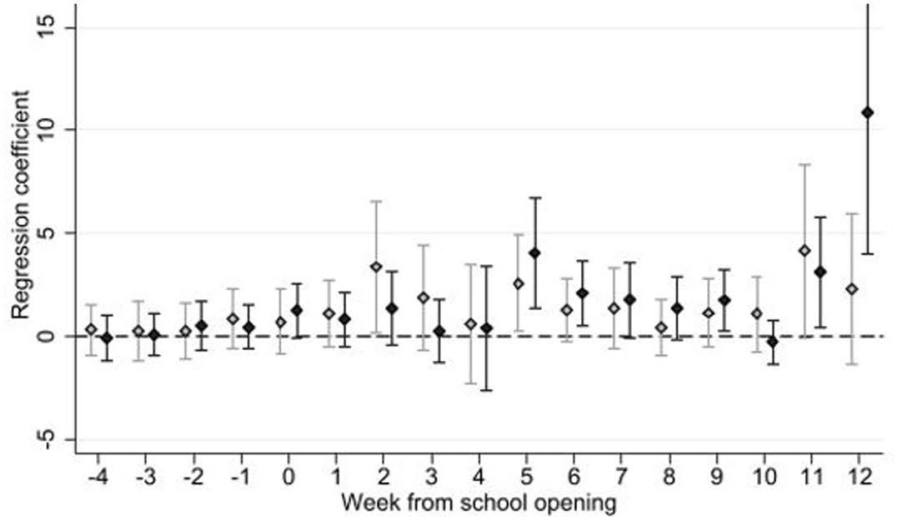

(d) South

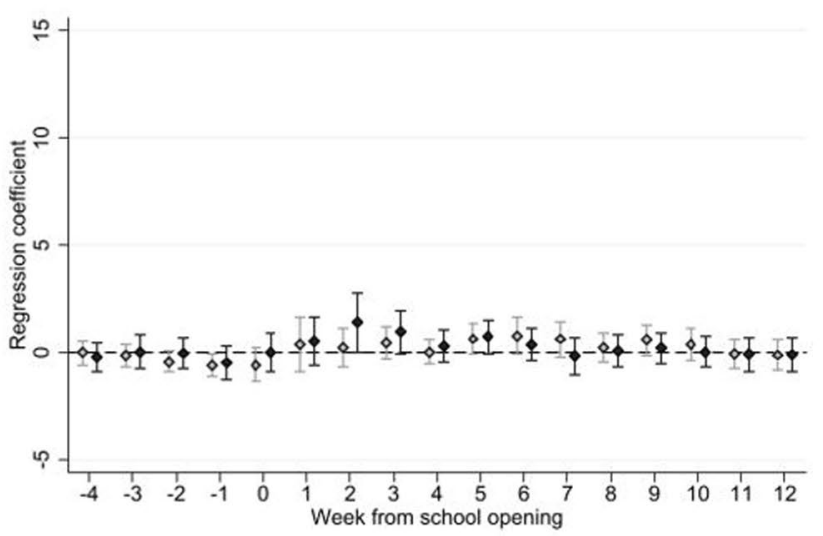

$\diamond$ Hybrid $\bullet$ Traditional

Extended Data Fig. 6 | Adjusted absolute difference between SARS-CoV-2 cases among 10-19 year olds in counties with hybrid and traditional school modes relative to virtual for each week. Week 0 is the week in which school started for each county, stratified by region restricted to individuals age 10-19. Results were generated from multivariable Poisson regressions with robust standard errors. Black markers indicate traditional school mode and gray markers indicate hybrid school mode. Whiskers for each marker depict the upper and lower bounds of the $95 \%$ confidence interval ( $N=1,438$ for $\mathrm{NE}, 608$ for W, 1,791 for MW, and 2,504 for S). Google mobility data were not available for Week 12 following school opening in the Northeast, thus results are presented for the 11 weeks following school opening in that region (panel A). 


\section{(a) Northeast}

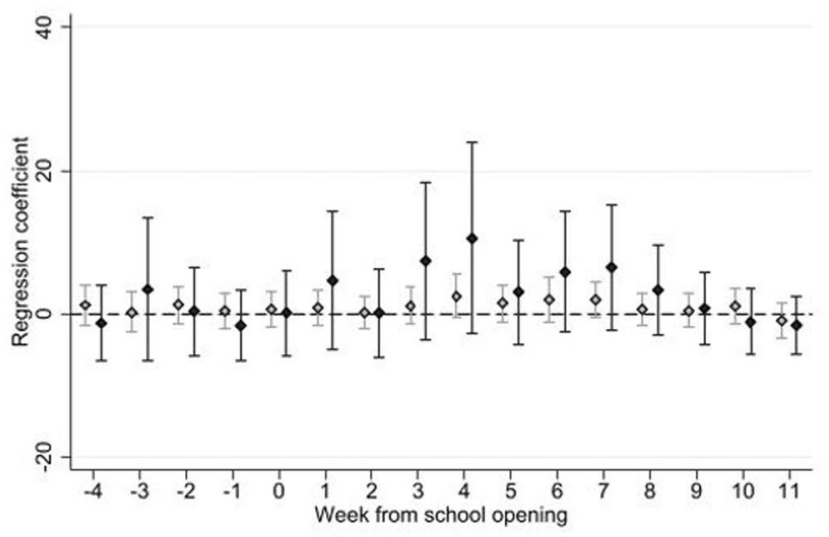

(c) Midwest

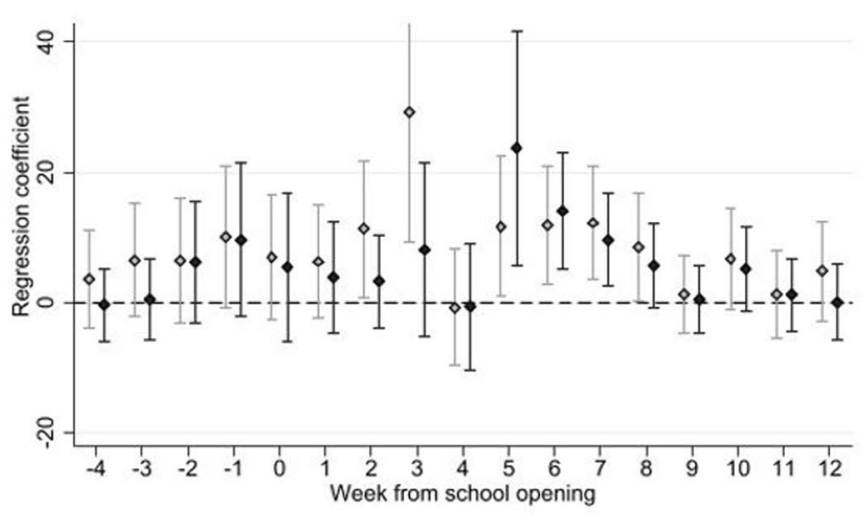

\section{(b) West}

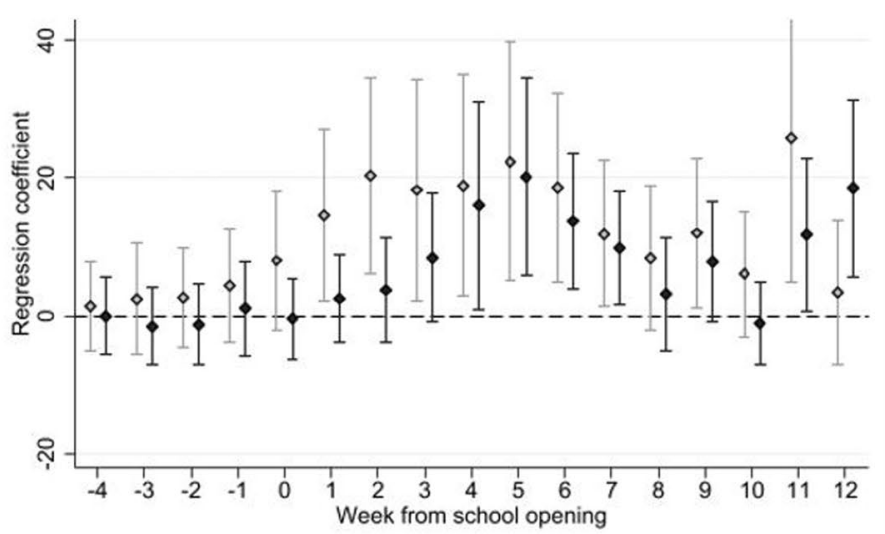

(d) South

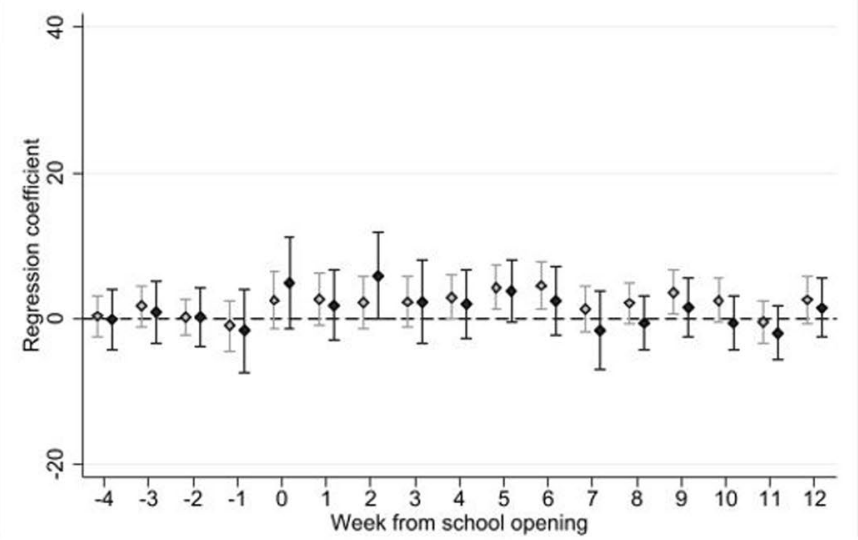

$\diamond$ Hybrid * Traditional

Extended Data Fig. 7 | Adjusted absolute difference between SARS-CoV-2 cases among adults in counties with hybrid and traditional school modes relative to virtual for each week. Week 0 is the week in which school started for each county, stratified by region restricted to individuals age $20+$. Results were generated from multivariable Poisson regressions with robust standard errors. Black markers indicate traditional school mode and gray markers indicate hybrid school mode. Whiskers for each marker depict the upper and lower bounds of the $95 \%$ confidence interval ( $N=1,467$ for $N E, 623$ for $\mathrm{W}, 1,852$ for $\mathrm{MW}$, and 2,709 for S). 
Extended Data Table 1 | Descriptive statistics of independent variables at county level on the week of school opening

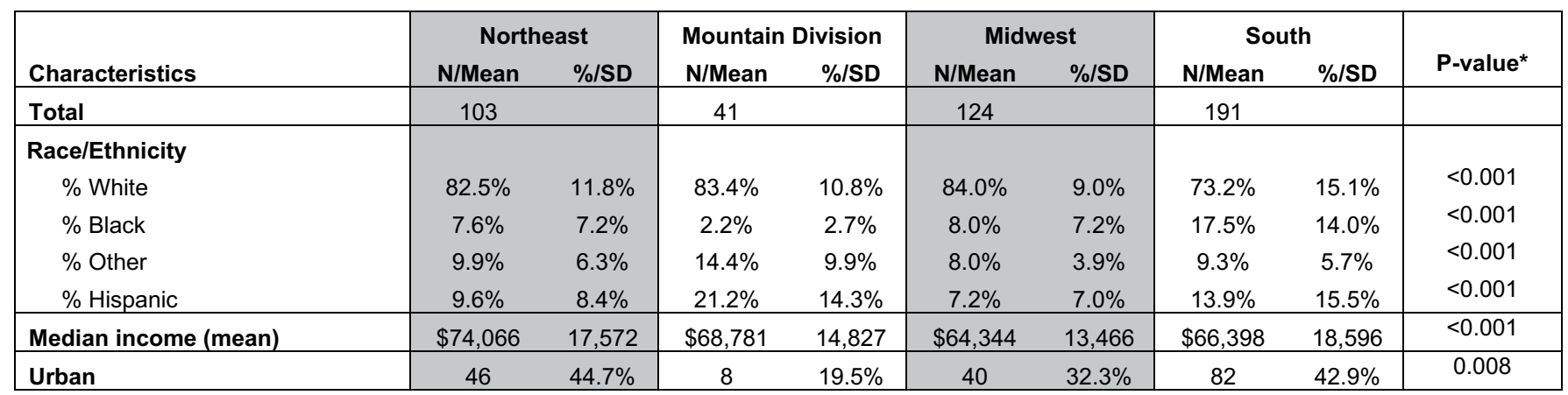

* P-values were obtained from one-way analysis of variance models. 


\section{Reporting Summary}

Nature Research wishes to improve the reproducibility of the work that we publish. This form provides structure for consistency and transparency in reporting. For further information on Nature Research policies, see our Editorial Policies and the Editorial Policy Checklist.

\section{Statistics}

For all statistical analyses, confirm that the following items are present in the figure legend, table legend, main text, or Methods section.

$\mathrm{n} / \mathrm{a}$ Confirmed

$\bigotimes$ The exact sample size $(n)$ for each experimental group/condition, given as a discrete number and unit of measurement

Х $\square$ A statement on whether measurements were taken from distinct samples or whether the same sample was measured repeatedly

The statistical test(s) used AND whether they are one- or two-sided

Only common tests should be described solely by name; describe more complex techniques in the Methods section.

A description of all covariates tested

A description of any assumptions or corrections, such as tests of normality and adjustment for multiple comparisons

A full description of the statistical parameters including central tendency (e.g. means) or other basic estimates (e.g. regression coefficient)

AND variation (e.g. standard deviation) or associated estimates of uncertainty (e.g. confidence intervals)

For null hypothesis testing, the test statistic (e.g. $F, t, r$ ) with confidence intervals, effect sizes, degrees of freedom and $P$ value noted Give $P$ values as exact values whenever suitable.

Х $\square$ For Bayesian analysis, information on the choice of priors and Markov chain Monte Carlo settings

$\bigotimes \square$ For hierarchical and complex designs, identification of the appropriate level for tests and full reporting of outcomes

$\triangle \square$ Estimates of effect sizes (e.g. Cohen's $d$, Pearson's $r$ ), indicating how they were calculated

\section{Our web collection on statistics for biologists contains articles on many of the points above.}

\section{Software and code}

Policy information about availability of computer code

Data collection No software was used for data collection. School reopening mode data were obtained from Burbio, which includes manually-validated information from 1,200 school districts across the US, representing approximately 35,000 schools in 50 states, and $47 \%$ of student enrollment in public K-12 schools. Incident cases of SARS-CoV-2 per day at a county level were obtained from the Centers for Disease Control and Prevention (CDC) dataset. Data about community-level mitigation measures were obtained through the Oxford University dataset, which contain data about federal, state, and sub-state policies. Community Mobility Data were accessed from the Google Movements dataset.

Data analysis All analyses were completed using STATA version 16 (methods section). Code is available for review on the Harvard Dataverse, link here: https://dataverse.harvard.edu/dataverse/school-opening-covid).

For manuscripts utilizing custom algorithms or software that are central to the research but not yet described in published literature, software must be made available to editors and

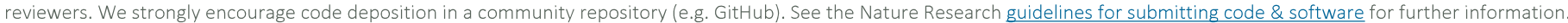

\section{Data}

Policy information about availability of data

All manuscripts must include a data availability statement. This statement should provide the following information, where applicable:

- Accession codes, unique identifiers, or web links for publicly available datasets

- A list of figures that have associated raw data

- A description of any restrictions on data availability

Data underlying this manuscript were collected from a variety of sources. Community Mobility Report data from Google and the COVID-19 Government Response Tracker data from Oxford University are publicly available for download. Data from Burbio, LLC are available via purchase agreement and data use agreement with the company. Data from the CDC restricted access dataset are available via data use agreement with the CDC. 


\section{Field-specific reporting}

Please select the one below that is the best fit for your research. If you are not sure, read the appropriate sections before making your selection.
Life sciences
Behavioural \& social sciences
Ecological, evolutionary \& environmental sciences

For a reference copy of the document with all sections, see nature.com/documents/nr-reporting-summary-flat.pdf

\section{Behavioural \& social sciences study design}

All studies must disclose on these points even when the disclosure is negative.

Study description

Research sample

Sampling strategy

Data collection

Timing

Data exclusions

Non-participation

Randomization
For this national retrospective cohort study that evaluated the impact of school opening mode (categorical variable) on community case rates of SARS-CoV-2 (quantitative variable), data were extracted from several data sources. School opening date and mode were collected from the Burbio school tracker, which tracks school openings in a sample of school districts across the US. Incidence of SARS-CoV-2 and COVID-19 related deaths were obtained from the CDC. Community-level SARS-CoV-2 mitigation measures were obtained from the Oxford University COVID-19 Government Response Tracker. Data were analyzed using an event-study framework.

The dataset included school districts with opening mode and date available for analysis in the Burbio dataset, which includes manually validated data about school opening mode and date from across the US. After excluding the Pacific region due to lack of variation in school mode, the complete dataset included 459 counties representing 895 public school districts in the US, which where then stratified by US census region. These districts represent approximately 5 half of all public school enrollment in the US.

School reopening mode data were obtained from Burbio, which includes manually-validated information from 1,200 school districts across the US, representing approximately 35,000 schools in 50 states, and $47 \%$ of student enrollment in public $\mathrm{K}-12$ schools. Burbio reviews a sample of districts representative of public school enrollment across the United States. Public school districts are classified into type of school mode, including traditional, defined as students participating in in-person learning $\geq$ four days per week; hybrid, defined as students divided into cohorts and attending school in-person two to three days per week; and virtual, defined as students attending school in a fully remote mode with no live, in-person instruction. Data available in the Burbio dataset include the date the school district opened and the proportion of schools that opened in each of the three different learning modes, stratified by school type (e.g., elementary, defined as kindergarten-5th grade, middle school, defined as 6th-8th grade, and high school, defined as 9 th-12th grade). The primary study question was the impact of school mode on community case rates of infection after school reopening and the Burbio dataset includes this information. These data were then linked to community case rates obtained directly from the CDC.

Data were obtained directly from existing databases, which were then linked together. The main exposure variable of interest was obtained from Burbio, which conducts manual review of a representative sample of districts across the US for school mode and opening date. Case rates, the main outcome measure, were obtained from the CDC. Community movement was collected from the Google movements dataset, which collects activity data based on user movement history. The Oxford dataset contains data about mitigation measure policies. Google movements and Oxford datasets were used as co-variates in the model, to adjust for factors potentially confounding the relationship between school opening and community case rates.

July-September, 2020

The Pacific division was excluded due to lack of variation in school mode.

This was a retrospective cohort study, therefore non-participation is not applicable.

This was a non-randomized retrospective cohort study.

\section{Reporting for specific materials, systems and methods}

We require information from authors about some types of materials, experimental systems and methods used in many studies. Here, indicate whether each material, system or method listed is relevant to your study. If you are not sure if a list item applies to your research, read the appropriate section before selecting a response. 
$\mathrm{n} / \mathrm{a}$ Involved in the study $\mathrm{n} / \mathrm{a}$ Involved in the study

\ $\square$ Antibodies

Х $\square$ ChIP-seq

$\square$ Eukaryotic cell lines

Х $\square$ Flow cytometry

Х $\square$ Palaeontology and archaeology

X $\square$ MRI-based neuroimaging

\ Animals and other organisms

\ $\square$ Human research participants

\ $\square$ Clinical data

$\bigotimes \square$ Dual use research of concern 